CERN-TH.6791/93

S.I.S.S.A. $11 / 93 /$ EP

\title{
EFFECTIVE ACTION AND ALL-ORDER GRAVITATIONAL EIKONAL AT PLANCKIAN ENERGIES
}

\author{
D. Amati \\ International School for Advanced Studies - Trieste \\ and INFN - Sezione di Trieste, Italy \\ M. Ciafaloni \\ Dipartimento di Fisica, Università di Firenze \\ and INFN - Sezione di Firenze, Florence, Italy \\ and \\ G. Veneziano \\ CERN - Geneva - Switzerland
}

\begin{abstract}
Building on previous work by us and by Lipatov, we present an effective action approach to the resummation of all semiclassical (i.e. $O\left(\hbar^{-1}\right)$ ) contributions to the scattering phase arising in high energy gravitational collisions. By using an infrared safe expression for Lipatov's effective action, we derive an eikonal form of the scattering matrix and check that the superstring amplitude result is reproduced at first order in the expansion parameter $R^{2} / b^{2}$, where $R, b$ are the gravitational radius and the impact parameter, respectively. If rescattering of produced gravitons is neglected, the longitudinal coordinate dependence can be explicitly factored out and exhibits the characteristics of a shock wave metric while the transverse dynamics is described by a reduced two-dimensional effective action. Singular behaviours in the latter, signalling black hole formation, can be looked for.
\end{abstract}

CERN-TH.6791/93

S.I.S.S.A. 11/93/EP

January 1993 


\section{Introduction}

In a series of papers ${ }^{(1,2)}$ we have advocated that, as a consistent theory of quantum gravity, string theory is able not only to reproduce Einstein's classical General Relativity at large distances, but also to provide systematic, calculable corrections to the leading order approximation.

The Gedanken experiment we conceived in order to substantiate this idea consists of the collision of light (or massless) particles at energies (much) larger than the Planck scale, i.e. in an environment in which gravity becomes a strongly interacting theory.

At sufficiently large distances (impact parameters) the dominant loop diagrams can be evaluated and resummed in closed form and simply yield an elastic eikonal amplitude corresponding to the leading order gravitational deflection predicted by General Relativity. This result can be (and has been) also obtained by other methods ${ }^{(3,4)}$.

At extremely high energies the most important corrections to the leading term have still the form of semiclassical contributions (i.e. of terms $O\left(\hbar^{-1}\right)$ in the phase) and intervene at distances that are still much larger than the intrinsic (quantum) scale of string theory. So far, they have only been computed to the first non-leading order ${ }^{(2)}$.

The nature of these corrections suggests that, after a regime of large deflection angles and copious particle production at intermediate impact parameters, a regime of gravitational collapse will set in below a critical angular momentum (for given centre-of-mass energy). This fits well with results obtained in the study of the gravitational collapse of large, continuous systems (such as a rotating star). In particular, numerical studies ${ }^{(5)}$ show that, below a critical value of $J / G M^{2}$ of order 1 , a finite fraction of the original mass collapses to a black hole, while the remaining fraction escapes at infinity.

General relativity studies closer to ours have been pursued by D'Eath and Payne (6) in their approach to black-hole collisions at small impact parameters. So far their results are also in agreement with the above picture.

In order to check the conjecture that gravitational collapse occurs also in the scattering of two light particles, a calculation of semiclassical terms to all orders is necessary (and hopefully sufficient). This is already a formidable task. Indeed, besides the general complexity of higher loop diagrams in string or field theory, the occurrence of infrared divergences complicates the analysis further. Although a Block-Nordsiek reinterpretation is possible ${ }^{(2)}$, a delicate treatment is necessary before being able to extract finite answers for infrared-safe quantities, such as the real part of the phase shift at non-leading order.

In this paper we propose an alternative approach based on an effective action tech- 
nique. The explicit computation of Ref. (2) allows us to identify the relevant modes that must enter the effective action. This means both the (momentum-dependent) producedgraviton polarization with its emission vertex ${ }^{(7,8)}$ and the modes responsible for its rescattering in the asymptotic regime in question.

The effective Lagrangian generated in this way is analogous to the one recently proposed by Lipatov ${ }^{(9)}$. We shall argue here that, with a proper infrared regularization, this Lagrangian is indeed suitable for a direct evaluation of the semiclassical scattering matrix: by solving the equations of motion that follow from such effective action one generates the generalized gravitational eikonal.

In this new language, the correct treatment of infrared (IR) singularities determines the correct form of the effective Lagrangian by fixing some total derivative, IR-sensitive (boundary) terms.

After having discussed the eikonal expansion in section 2 and the effective action in section 3, we shall tackle, in section 4, the simpler case in which rescattering is neglected. We will then be able to actually solve for the longitudinal dependence of all classical fields thus reducing the problem to a transverse dynamical effective action that is automatically IR safe.

The general form of the solution explicitly displays two longitudinal shock-wave metrics, with non-trivial transverse components in between the two wave fronts. The transverse field, as well as the profile function of the shock waves, is determined by a reduced effective action in the two transverse coordinates. We are then able to check that the perturbative solution of the equations of motion gives rise to a first non-leading eikonal contribution to the scattering amplitude that coincides with the one computed in Ref. (2) in a diagrammatic manner.

Of course, the final objective of the present approach is not the perturbative development it resums, but the study of non-perturbative solutions that may uncover new classical phenomena generated by a consistent treatment of quantum gravity; a study that we are now ready to tackle.

\section{The eikonal expansion}

Let us start recalling the gravitational scattering regime that we investigate ${ }^{(1)}$, and the related length scales. In string-gravity, the fundamental scale is the string length $\lambda_{s} \equiv \sqrt{\alpha^{\prime} \hbar}$, in terms of which the Planck length $\lambda_{p}$ (and thus the Newton constant $G$ ) is 
expressed in four uncompactified space-time dimensions as

$$
\sqrt{G \hbar}=\lambda_{p}=g \lambda_{s}
$$

where $g$ is the string loop expansion parameter, assumed to be small.

A very important scale in high-energy gravitational scattering turns out to be the classical (Schwarzschild) radius associated with the centre-of-mass energy of the collision:

$$
R \equiv 4 G E=2 G \sqrt{s}
$$

At (super) Planckian energies such a radius is always (much) larger than $\lambda_{p}$ and of the Compton wavelength of the colliding particles:

$$
G E^{2}>(>>) \hbar, \Rightarrow R>(>>) \hbar / E, \lambda_{p}
$$

In the case of string-gravity, physics also depends crucially on the ratio:

$$
\frac{R}{\lambda_{s}} \sim g \sqrt{\frac{G E^{2}}{\hbar}}
$$

which, because of the smallness of $g$, can be smaller or greater than 1 even at high energy.

For $R<\lambda_{s}$, string effects ${ }^{(1)}$ soften gravity at short distances and confirm the validity of a generalized uncertainty relation ${ }^{(10)}$ for the distance $\Delta x$ actually explored at a given momentum transfer $\Delta p$ :

$$
\Delta x>\frac{\hbar}{\Delta p}+\alpha^{\prime} \Delta p>\lambda_{s}
$$

By contrast, for $R>\lambda_{s}$, new semiclassical phenomena ${ }^{(2)}$ that can extend much beyond the string length do appear. It is to this interesting regime that we shall restrict our attention in this paper.

In the impact parameter representation, the amplitude is given, for $b \gg R$, by the leading eikonal approximation, corresponding to the resumation of all powers of $G E^{2}$ due to multigraviton exchanges. It has the form ${ }^{(1,3,4)}$

$$
\begin{gathered}
S(b, E)=\exp 2 i \delta_{0}(b, E), \\
\delta_{0}(b, E)=-\frac{G s}{\hbar} \log \lambda b, \quad s=4 E^{2},
\end{gathered}
$$

where $\lambda$ is an infrared cut-off related to the well-known infinite Coulomb phase. The Einstein deflection angle is given by the stationarity condition on the phase of the scattering wave:

$$
\theta_{c l}=-\frac{2 \hbar}{E} \frac{\partial \delta_{0}}{\partial b}=\frac{2 R}{b}=\frac{8 G E}{b} .
$$


We can interpret the leading eikonal expression (2.6) as a multiple scattering series, in which the (small) deflection angle -corresponding to a possibly large momentum transfer $t-$ is built up by many small momentum transfer graviton-exchange processes (cf. Fig. 1, $\left.t_{s} \ll \hbar / \lambda_{s}\right)$.

For $b$ approaching $R$, the subleading terms, suppressed by powers of $R / b$, become sizeable. We have shown in Ref. (2) that -at least up to two loops- the $S$-matrix admits a generalized unitary eikonal representation. In the elastic channel

$$
S_{e l}=\exp 2 i\left(\delta_{0}+\delta_{1}+\delta_{2}+\cdots\right)
$$

where $\delta_{0}$ is given by Eq. (2.6) and, for pure gravity ${ }^{(2)}$,

$$
\begin{gathered}
\delta_{1}(b, E)=\frac{6}{\pi} \frac{G^{2} s}{b^{2}} \log s \\
\delta_{2}(b, E)=\frac{2 G^{3} s^{2}}{\hbar b^{2}}\left[1+\frac{2 i}{\pi} \log s(-\log b \lambda+1)\right] .
\end{gathered}
$$

Notice that $\delta_{1}$ is purely real. The imaginary part of $\delta_{2}$ is expected from unitarity, because of graviton bremsstrahlung predicted by the inelastic amplitudes at the corresponding order. Therefore, the appearance of the infrared cut-off $\lambda$ in $\operatorname{Im} \delta_{2}$ is due to soft graviton emission and thus admits a Bloch-Nordsieck interpretation consistent with a finite value for $\operatorname{Re} \delta_{2}$. This extra contribution to the phase modifies the Einstein deflection angle:

$$
\sin \frac{\theta_{c l}}{2}=\frac{R}{b}\left(1+\frac{R^{2}}{b^{2}}+\ldots\right)
$$

The one-loop correction $\delta_{1}$ in Eq. (2.9) is a typical quantum effect (of order $\hbar$ with respect to $\left.\delta_{0}\right)$ and is irrelevant in our regime $R \gg \lambda_{s}>\lambda_{p}$. Thus we shall concentrate on $\delta_{2}$ and on similar semiclassical corrections, i.e. those contributions to the eikonal that - as the leading one- are of order $\hbar^{-1}$ at fixed "charge" Gs, and contain arbitrary powers of $R^{2} / b^{2}$.

The result for $\delta_{2}$ was obtained in Ref. (2) by computing the two-loop diagram of Fig. 2, where the dashed lines represent string-gravitons or, in an equivalent language, ReggeGribov gravitons. In terms of graviton fields (wavy lines) the hybrid vertex occurring in Fig. 2 corresponds to a sum of Feynman diagrams (Fig. 3) where gravitons are emitted off internal and external legs.

The explicit expression for the emission amplitude has the form ${ }^{(7,8)}$

$$
A_{\mu \nu}=\frac{\kappa^{2} s^{2}}{q_{1}^{2} q_{2}^{2}} \kappa J_{\mu \nu}, \quad \kappa^{2}=8 \pi G,
$$


in terms of the conserved and traceless vertex function $J_{\mu \nu}$, given by

$$
J_{\mu \nu}=\frac{\eta_{\mu} \eta_{\nu}}{\mathbf{k}^{2}} \frac{2}{i}\left(\left|q_{1}\right|^{2}\left|q_{2}\right|^{2}-q_{1}^{2} q_{2}^{*^{2}}\right)+c . c .
$$

Here we have introduced the complex transverse components

$$
q=q^{1}+i q^{2}, \quad q^{*}=q^{1}-i q^{2}
$$

for the momentum transfers $q_{i}^{\mu}(i=1,2)$ of Fig. 3 , and the complex polarization vector

$$
\eta^{\mu}=\frac{1}{\sqrt{2}}\left(\varepsilon_{L}^{\mu}(k)+i \varepsilon_{T}^{\mu}(k)\right), \quad \varepsilon_{L} \cdot k=\varepsilon_{T} \cdot k=0, \quad \varepsilon_{L}^{2}=\varepsilon_{T}^{2}=-1,
$$

in which $\varepsilon_{L}\left(\varepsilon_{T}\right)$ are 4-vectors parallel (perpendicular) to the incoming beam direction.

The emission amplitude $A_{\mu \nu}(1,2)$ was sewn up to its absorption analogue $A^{\mu \nu^{*}}(3,4)$ in Ref. (2) in order to compute $\operatorname{Im} \delta_{2}$ (see Fig. 2); Re $\delta_{2}$ was then computed by using analyticity and unitarity arguments for the full $S$-matrix, and by actually witnessing the ensuing cancellation of infrared divergences. The extension of this rigorous procedure to higher loops in order to compute $\operatorname{Re} \delta_{4}$, etc. looks too cumbersome to be tractable. We shall then propose a shortcut procedure, by checking it later at the level of Re $\delta_{2}$.

The idea is simple: infrared divergences represent a trouble related, of course, to the physical problem of soft bremsstrahlung. Their elimination should not disturb the computation of the real part of the eikonal, whose structure is still provided by the diagram in Fig. 2. Thus we shall define our effective eikonal function by sewing up the vertices $A^{\mu \nu}$ by an infrared-safe prescription. At the two-loop level, this amounts to defining, from Eq. (2.9), a regularized amplitude

$$
\begin{gathered}
\delta_{2}-\delta_{2_{I R}}=\frac{1}{s}\left\langle A_{\mu \nu}(1,2) A^{\mu \nu}(3,4)\right\rangle_{r e g}=\operatorname{Re} \delta_{2}\left(1+\frac{2 i}{\pi} \log s\right), \\
\operatorname{Re} \delta_{2}=\frac{1}{2} \frac{G s}{\hbar} \frac{R^{2}}{b^{2}}, \quad R=2 G \sqrt{s},
\end{gathered}
$$

with a subtraction procedure to be described in detail in Section 4.

With this regularization, one can define an effective $S$-matrix that exponentiates the real part of the regularized $H$-diagram (Fig. 2) in addition to the single-graviton exchange diagram (Fig. 1):

$$
S_{e f f}(b, E)=\exp 2 i\left(\delta_{0}(b, E)+\operatorname{Re} \delta_{2}(b, E)+\ldots\right)
$$

The resulting expression admits again the multiple scattering interpretation given before, where, however, two kinds of irreducible interactions now take place. 


\section{The effective action}

We have identified in Ref. (1) the irreducible diagrams that generate all relevant semiclassical corrections to the eikonal. They are subleading by one power of $s$ per loop pair and come from the exchange of $n$ upper and $n$ lower Regge-Gribov gravitons, interacting at tree level as described in Fig. 4. Their order of magnitude is given by

$$
\left(\frac{G s}{\hbar}\right)^{n+1}\left(\frac{G \hbar}{b^{2}}\right)^{n} \propto \frac{G s}{\hbar}\left(\frac{R^{2}}{b^{2}}\right)^{n}
$$

so that they represent the irreducible semiclassical contributions mentioned before, which are down by $\left(R^{2} / b^{2}\right)^{n}$ with respect to the leading eikonal.

The semiclassical $S$-matrix of Eq. (2.16) is expected to come from exponentiating the sum of such properly regularized irreducible diagrams, which defines the generalized eikonal function we are looking for.

In order to implement explicitly this exponentiation, let us first notice that the graviton couplings to the external particle lines in Fig. 4 can be replaced by the purely eikonal ones $\left(\propto p_{\mu} p_{\nu}\right)$, which, by definition, disregard recoil effects. Such effects, involving the momentum transfers of the exchanged gravitons, yield subleading corrections to the eikonal vertices (cf. Fig. 3c) which have already been included in the emission vertex $J_{\mu \nu}$ of Eq. (2.12) and are thus taken into account in the tree amplitude of Fig. 4.

The above remark implies that the external particle lines can be represented as classical sources of the graviton field $h^{\mu \nu}(x)$

$$
T_{\mu \nu} h^{\mu \nu}=T_{--}(x) h^{--}(x)+T_{++}(x) h^{++}(x)
$$

through their energy momentum tensor $T_{\mu \nu}$ whose non-zero components are:

$$
\begin{gathered}
T_{--}=\kappa E \delta\left(x^{-}\right) \delta^{2}(\mathbf{x}), \quad T_{++}=\kappa E \delta\left(x^{+}\right) \delta^{2}(\mathbf{x}-\mathbf{b}), \\
\left(x^{ \pm}=x^{0} \pm x^{3}, \quad \mathbf{x}=\left(x^{1}, x^{2}\right)\right) .
\end{gathered}
$$

Calling $L_{e f f}\left(h_{\mu \nu}\right)$ the effective Lagrangian generating the irreducible tree amplitudes of Fig. 4, the scattering matrix embodying all iterations of such irreducible diagrams (leading and subleading) will be given by

$$
S_{e f f}(b, E)=\left\langle\exp \frac{i}{\hbar} A\left(h_{\mu \nu}\right)\right\rangle_{t r e e}=\exp \left[\frac{i}{\hbar} A\left(h_{c l}^{\mu \nu}\right)\right]
$$


where

$$
A\left(h^{\mu \nu}\right)=\int d^{4} x\left(L_{e f f}\left(h^{\mu \nu}\right)+T_{\mu \nu} h^{\mu \nu}\right)
$$

and $h_{c l}^{\mu \nu}$ represents the solution of the classical equations of motion that minimize $A$ with the sources $T^{\mu \nu}$ provided by Eq. (3.3). Thus $\frac{1}{2} A\left(h_{c l}^{\mu \nu}\right)$ represents the generalized eikonal function.

Lipatov $^{(9)}$ has proposed an explicit form for $L_{e f f}$ justified in the high-energy regime in which all subenergies among initial and emitted gravitons are large. Despite the present lack of complete derivation of an effective action from first principles, we think that for the computation of the semiclassical $S$-matrix in Eq. (3.4) the Lipatov form can safely be used. The reason for this lies in the very fact that the diagrams in Fig. 4 and their iterations never allow internal graviton loops, whose regularization would probably require again string gravity. A check of our conjecture will be the agreement of the effective action method with our previous result for $\operatorname{Re} \delta_{2}$, at first subleading level.

Lipatov's effective Lagrangian contains not only the emission vertex $J_{\mu \nu}$ illustrated before (Eq. 2.12), but also a rescattering of the emitted graviton with external particles through eikonal vertices $\left(\propto k_{\mu} k_{\nu}\right)$. In the diagrammatic representation of Fig. 3, this implies the two vertices of Fig. 5 .

Note that $J_{\mu \nu}$ in Eq. (2.12) emits a graviton with a well-defined, although momentum dependent, polarization $\eta_{\mu \nu}=\operatorname{Im}\left(\eta_{\mu} \eta_{\nu} \hat{q}_{1} \hat{q}_{2}^{*}\right)$. This polarization -in the high-energy regime in question- will be preserved in the subsequent interactions of that graviton with the external particles. This is why there is a single rescattering vertex in Fig. 5.

The above discussion indicates that the relevant components of the gravitational field that will enter the effective Lagrangian are the longitudinal ones $h^{++}, h^{--}$(represented by dashed lines in Figs. 4 and 5, emitted each by one of the external particles) and the intermediate (complex) component

$$
\begin{gathered}
h_{\eta \eta}=h_{\mu \nu} \eta^{\mu} \eta^{\nu}=2 \frac{\partial^{2}}{\partial z^{*^{2}}} \phi\left(z, z^{*}, x^{+}, x^{-}\right), \\
\left(z=x^{1}+i x^{2}, x^{ \pm}=x^{0} \pm x^{3}\right)
\end{gathered}
$$

represented by wavy lines in Figs. 4 and 5 .

The presence of the transverse propagator $\left(\mathbf{k}^{2}\right)^{-1}$ in $J_{\mu \nu}$ of Eq. (2.12) shows the nonlocality of the effective Lagrangian in terms of the graviton field $h_{\mu \nu}$. This non-locality is avoided by expressing $h_{\eta \eta}$ in terms of the complex scalar field $\phi$ as in Eq. (3.6), at the price, of course, of higher derivatives in the $\phi$ "kinetic" term. 
Indeed Lipatov's expression of $L_{e f f}$ in terms of $h^{++}, h^{--}$and $\phi$ reads

$$
L_{e f f}=L_{0}+L_{e}+L_{r}
$$

where

$$
\begin{gathered}
L_{0}=-2 \partial h^{--} \partial^{*} h^{++}+\partial_{+} \partial^{*^{2}} \phi \partial_{-} \partial^{2} \phi^{*}, \\
\left(\partial=\frac{\partial}{\partial z}, \partial^{*}=\frac{\partial}{\partial z^{*}}, \partial_{ \pm}=\frac{\partial}{\partial x^{ \pm}}\right)
\end{gathered}
$$

is the kinetic term,

$$
\begin{gathered}
L_{e}=J(x) \phi^{*}(x)+J^{*}(x) \phi(x), \\
J(x)=4 \kappa\left[\partial^{*}{ }^{2} h^{++} \partial^{2} h^{--}-\partial^{*} \partial h^{++} \partial^{*} \partial h^{--}\right],
\end{gathered}
$$

is the graviton emission-absorption term corresponding to the momentum-dependent vertex $J_{\mu \nu}$ of Eq. (2.12), and

$$
L_{r}=2 \kappa\left(h^{++} \partial^{*^{2}} \phi^{*} \partial_{+} \partial_{+} \partial^{2} \phi+h^{--} \partial^{2} \phi^{*} \partial_{-} \partial_{-} \partial^{*^{2}} \phi\right)
$$

is the rescattering term, where the eikonal vertices $\sim k_{ \pm}^{2}$ appear.

We note here that, following Lipatov, the kinetic term for $h_{\eta \eta}$ contains only the longitudinal derivatives $\partial_{ \pm}$and not the transverse ones. We think that this simplification is plausible for the calculation of the real part of the eikonal, because the longitudinal graviton momenta vary up to $E$ and are thus on the average much larger than the transverse ones, of order $\hbar / b$. This would not be true for the imaginary part, in which the mass-shell condition forces longitudinal and transverse momenta to be of the same order. By the same token, the real part of the longitudinal propagators of $\phi$ will be evaluated in the following with a principal value prescription, as a remainder of the Feynman prescription for the full propagator.

The identification of longitudinal $\left(h^{++}, h^{--}\right)$and intermediate $\left(\phi, \phi^{*}\right)$ components as the relevant degrees of freedom of the effective Lagrangian recalls the attempt by $E$. and H. Verlinde' (11) at separating longitudinal and transverse degrees of freedom by a gauge choice. The non-trivial difference lies in the fact that the definition of the intermediate component $h_{\eta \eta}$ is momentum-dependent, thus not purely transverse to the incoming beam and that, as said before, in terms of $h_{\eta \eta}$ the effective vertices are non-local. Furthermore, only in the non-interacting limit $\left(\frac{R}{b} \rightarrow 0\right)$ that gives the leading eikonal is the decoupling of longitudinal and transverse components possible, as advocated in Ref. (11).

Let us stress again that the aforementioned non-locality has a very physical origin. It is indeed due to the recoil effects of the external particles that induce a transverse propagator 
factor $\left(\mathbf{k}^{2}\right)^{-1}$ in the vertex $J_{\mu \nu}$, as discussed before. Thus $L_{e f f}$ incorporates graviton modifications to the external particle propagation, in addition to the purely gravitational interactions of intermediate gravitons (cf. again Fig. 3).

\section{Solutions without rescattering}

From the effective action in Eqs. (3.5) and (3.8)-(3.10) it is possible to obtain classical solutions and then compute the semiclassical $S$-matrix from the expression in Eq. (3.4). We shall address, however, a simplified problem, which is obtained by neglecting the rescattering term $L_{r}$ in the effective Lagrangian. Let us note that this simplification does not affect our first goal, which is to check the result for $\operatorname{Re} \delta_{2}$ obtained previously ${ }^{(2)}$, because the $H$-diagram in Fig. 2 does not contain rescattering vertices. Furthermore, this simplification allows us to identify a general class of solutions for which the infrared-safe definition of the action - which is anyway needed according to the discussion in Section 2can be performed in an explicit and simple way.

The general equations of motion derived from Eqs. (3.5) to (3.10) are

$$
\begin{gathered}
|\partial|^{2} h^{++}+2 \kappa\left[\partial^{2}\left(\phi^{*} \partial^{*^{2}} h^{++}\right)+\partial^{*^{2}}\left(\phi \partial^{2} h^{++}\right)-|\partial|^{2}\left[\left(\phi+\phi^{*}\right)|\partial|^{2}\left(h^{++}\right)\right]\right. \\
+\frac{\kappa E}{2} \delta\left(x^{-}\right) \delta^{2}(\mathbf{x})=-\kappa \partial^{*^{2}}\left(\phi^{*} \partial_{+}^{2} \partial^{2} \phi\right), \\
\partial_{+} \partial_{-}|\partial|^{4} \phi-2 \kappa\left(\partial^{*^{2}} h^{++} \partial^{2} h^{--}-|\partial|^{2} h^{++}|\partial|^{2} h^{--}\right)= \\
=-\kappa \partial^{*^{2}}\left(h^{++} \partial_{+}^{2} \partial^{2} \phi\right)-\kappa \partial^{2}\left(h^{--} \partial_{-}^{2} \partial^{*^{2}} \phi\right),
\end{gathered}
$$

with similar ones for $h^{--}$and $\phi^{*}$.

By dropping the rescattering terms, the r.h.s. of Eqs. (4.1), (4.2) are set to zero, and we realize that the longitudinal variable dependence can be factored out in the following solution: *.

$$
h^{++}=\kappa E \delta\left(x^{-}\right) a\left(z, z^{*}\right), \quad h^{--}=\kappa E \delta\left(x^{+}\right) \bar{a}\left(z, z^{*}\right)
$$

* The inversion of the $\partial_{+} \partial_{-}$operator in Eq. (4.2) for the $\phi$ field is here performed by a principal value prescription that is appropriate for our $S$-matrix evaluation, as discussed in Section 3. Using a retarded propagator would amount to replacing $\frac{1}{2} \theta\left(x^{+} x^{-}\right)$ by $\theta\left(x^{+}\right) \theta\left(x^{-}\right)$. In any case, we use a definition of the Heaviside function with $\theta(0)=\frac{1}{2}$. With this proviso, the form of Eq. (4.4) is uniquely determined. We have also checked that reintroducing transverse derivatives in the $\phi$ propagator does not modify the final result. 


$$
\begin{gathered}
\phi=\frac{\kappa}{2}(\kappa E)^{2} \frac{1}{2} \theta\left(x^{+} x^{-}\right) \varphi\left(z, z^{*}\right), \\
a^{*}\left(z, z^{*}\right)=a\left(z, z^{*}\right)=\bar{a}\left(b-z, b-z^{*}\right), \\
\varphi^{*}\left(z, z^{*}\right)=\varphi\left(b-z, b-z^{*}\right),
\end{gathered}
$$

which represents a shock-wave longitudinal metric (with profile functions $a$ and $\bar{a}$ ) and a non-trivial transverse metric -related to $\varphi$ by $\mathrm{Eq}(3.6)$ - in between the two wavefronts. Here $a, \bar{a}, \varphi$ satisfy a reduced set of equations of motion in the transverse variables $z=$ $x^{1}+i x^{2}$ and $z^{*}$ given by

$$
\begin{gathered}
\left(\partial^{*} \partial\right)^{2} \varphi=4\left(\partial^{*^{2}} a \partial^{2} \bar{a}-\partial^{*} \partial a \partial^{*} \partial \bar{a}\right) \equiv j(x), \\
\partial^{*} \partial a+(\pi R)^{2}\left[\partial^{2}\left(\varphi^{*} \partial^{*^{2}} a\right)+\partial^{*^{2}}\left(\varphi \partial^{2} a\right)-\partial^{*} \partial\left[\left(\varphi+\varphi^{*}\right) \partial^{*} \partial a\right)\right]=-\frac{1}{2} \delta(x) \\
\bar{a}(\mathbf{x})=a(\mathbf{b}-\mathbf{x}), \varphi^{*}(\mathbf{x})=\varphi(\mathbf{b}-\mathbf{x}),
\end{gathered}
$$

where we use also the $\mathbf{x}$ notation, if more convenient.

In the reduced equation (4.4) the gravitational radius $R=4 G E$ of the system appears explicitly and plays the role of coupling constant of the problem. For $R=0$ eq. (4.4) reduces to the free-field solution

$$
a^{(0)}=-\frac{1}{2 \pi} \log \frac{z z^{*}}{L^{2}}, \bar{a}^{(0)}(\mathbf{x})=a^{(0)}(\mathbf{b}-\mathbf{x}), \varphi^{(0)}=\varphi^{(0)^{*}}=0,
$$

where we have introduced the large-distance scale $L=\frac{1}{\lambda}$ playing the role of infrared cutoff, which fixes the scale of $a^{(0)}$ by the condition $a^{(0)}(L)=0$. Thus at the leading order the profile function of the longitudinal shock wave -and the absence of transverse componentscorresponds to an Aicheburg-Sexl ${ }^{(12)}$ shock-wave metric. Introducing (4.5) into Eq. (3.4) we obtain the leading eikonal $S$-matrix ${ }^{(13)}$ already derived in a variety of ways.

To go beyond this leading expression, let us go back to our solution (4.3) of the equations of motion. By replacing it into Eqs. (3.8)-(3.10) we obtain a reduced form of the action that involves transverse variables only and that we write in the following form

$$
\begin{aligned}
& A(a, \bar{a}, \varphi)=2 \pi G s\left[\bar{a}(0)+a(b)+\int d^{2} x\left(\bar{a} \partial^{*} \partial a++a \partial^{*} \partial \bar{a}+\right.\right. \\
& \left.\left.+\left(\pi \frac{R}{2}\right)^{2}\left(-\frac{1}{2} \varphi\left(\partial^{*} \partial\right)^{2} \varphi^{*}-\frac{1}{2} \varphi^{*}\left(\partial^{*} \partial\right)^{2} \varphi+\varphi^{*} j+j^{*} \varphi\right)\right)\right] .
\end{aligned}
$$

This form will now be argued to be infrared-safe.

In fact, the reduced action (4.6) differs from a naïve rewriting of the various terms in Eqs. (3.8) to (3.10) by total divergences, i.e. by boundary terms that leave the equations 
of motion invariant but could introduce infrared divergences due to the long-distance contributions. The particular form in Eq. (4.6) is characterized by the fact that all derivatives act on a single field and turns out to be infrared-safe because the behaviour of $\partial^{*} \partial a$ and $\left(\partial^{*} \partial\right)^{2} \varphi$, found from the equations of motion, is good enough to make all the integrals converge, as we shall see below.

In order to compute the $S$-matrix, we have to find the classical solutions of the reduced equations of motion (4.4) and then evaluate the classical action (4.6) so as to obtain, by $(3.4)$,

$$
S_{e f f}=\exp \left[\frac{i}{\hbar} A\left(a_{c l}, \bar{a}_{c l}, \varphi_{c l}\right)\right]
$$

For instance the leading eikonal function is recovered by setting $R=0$ in Eq. (4.6) and using the free-field solution (4.5)

$$
S^{(0)}=\exp 2 \pi i \frac{G s}{\hbar} \frac{1}{2}(\bar{a}(0)+a(b))=\exp \left(-2 \frac{i}{\hbar} G s \log \frac{b}{L}\right),
$$

in agreement with the value of $\delta_{0}$ quoted in Eq. (2.6).

To proceed further in the $\frac{R}{b}$ expansion of Eqs. (4.4) to (4.6) we need a more precise definition of the relevant solutions of the reduced equations of motion which, being a set of fourth-order differential equations, possess a lot of arbitrariness. To be definite, consider the first non-trivial iteration

$$
a=a^{(0)}+R^{2} a^{(1)}+\cdots, \quad \varphi=\varphi^{(1)}+\cdots
$$

and replace Eq. (4.9) in Eq. (4.4) to get

$$
\begin{gathered}
\left(\partial^{*} \partial\right)^{2} \varphi^{(1)}=4 \partial^{*^{2}} a^{(0)} \partial^{2} \bar{a}^{(0)} \\
\left(\partial^{*} \partial\right) a^{(1)}=-\pi^{2} \partial^{2}\left(\varphi^{*^{(1)}} \partial^{*^{2}} a^{(0)}\right)+\text { c.c. }
\end{gathered}
$$

where we have used the property that $a^{(0)}$, given in Eq. (4.5), is harmonic for $z \neq 0$ $\left(\partial^{*} \partial a^{(0)}=-\frac{1}{2} \delta^{2}(\mathbf{x})\right)$.

To start with, $a^{(1)}$ and $\varphi^{(1)}$ are defined up to solutions of the homogeneous equations

$$
\partial^{*} \partial \delta a^{(1)}=0, \quad\left(\partial^{*} \partial\right)^{2} \delta \varphi^{(1)}=0
$$

yielding

$$
\begin{gathered}
\delta a^{(1)}=f(z)+c . c . \\
\delta \varphi^{(1)}=g(z)+g\left(b-z^{*}\right)+z^{*} h(z)+(b-z) h\left(b-z^{*}\right),
\end{gathered}
$$


where $f, g$ and $h$ are arbitrary analytic functions. We shall determine them by the following requirements:

(a) $a^{(1)}$ and $\varphi^{(1)}$ should be single-valued outside the singularity points at $z=0$ and $z=b$.

(b) For $z \rightarrow 0, b, a^{(1)}$ and $\varphi^{(1)}$ should be as regular as possible, so as to avoid ultraviolet divergences in the action.

(c) The longitudinal field $a$ is normalized by $a(L) \simeq \bar{a}(L)=0$, where $L=1 / \lambda$ is the infrared scale of the problem.

Subject to the above conditions, we find the solutions

$$
\begin{gathered}
a^{(1)}=\frac{1}{4 \pi}\left[\frac{1}{|z|^{2}}\left|1-\frac{z}{b}\right|^{2} \log \left|1-\frac{z}{b}\right|^{2}+\frac{1}{b z}+\frac{1}{b z^{*}}-\frac{1}{b^{2}} \log \frac{L^{2}}{b^{2}}\right], \\
\varphi^{(1)}=\frac{1}{\pi^{2}}\left[\log \frac{z^{*}}{b} \log \left(1-\frac{z}{b}\right)+\frac{z}{b} \log \frac{z^{*}}{b}+\left(1-\frac{z^{*}}{b}\right) \log \left(1-\frac{z}{b}\right)+f\left(\frac{z}{b}\right)+f\left(1-\frac{z^{*}}{b}\right)\right], \\
\left.f(z) \equiv-\frac{1}{2}\left(\frac{\pi^{2}}{6}-1\right)+\int_{0}^{z} d x \frac{x}{x-1} \log x .14\right)
\end{gathered}
$$

Note first that $a^{(1)}$ and $\varphi^{(1)}$ are single-valued for $z \neq 0, b$ thanks to a judicious use of the additive arbitrary functions (4.12). For instance, the basic solution of Eq. (4.10) for $\varphi^{(1)}$, given by $\log \frac{z^{*}}{b} \log \left(1-\frac{z}{b}\right)$ (a product of analytic and anti-analytic functions) is not single-valued, but $\varphi^{(1)}$ in Eq. (4.14) instead is.

A similar remark holds for $z \rightarrow 0, b$, where the rather singular ultraviolet behaviour of the basic solutions is cancelled by the additive arbitrary functions of the form (4.12), which are thus fully determined. For instance, for $z \rightarrow 0$ we have the behaviour

$$
a^{(1)} \sim \operatorname{const}+\frac{1}{b^{2}}\left(\frac{z}{z^{*}}+\frac{z^{*}}{z}\right)+0\left(\frac{|z|}{b^{3}}\right), \quad \varphi^{(1)} \sim-\frac{1}{\pi^{2}}\left(\frac{z}{b}+\frac{z^{*}}{b}\right)+0\left(\frac{|z|^{2}}{b^{2}} \log |z|\right)
$$

in particular $\varphi^{(1)}(0)=0$, and similarly for $z \rightarrow b$,

$$
a^{(1)} \sim \text { const }+0\left(\frac{|z-b|}{b^{3}}\right), \quad \varphi^{(1)} \sim-\frac{1}{\pi^{2}}\left(1-\frac{z}{b}+1-\frac{z^{*}}{b}\right)+0\left(\frac{|b-z|^{2}}{b^{2}} \log |b-z|\right),
$$

while we also have, by Eq. (4.4),

$$
\begin{gathered}
\partial^{*} \partial a^{(1)}=\frac{1}{\pi} \frac{1}{|z|^{4}}\left(\log \left(1-\frac{z}{b}\right)+\frac{z}{b}+c . c .\right), \\
\left(\partial^{*} \partial\right)^{2} \varphi^{(1)}=\frac{1}{\pi^{2}} \frac{1}{z^{*^{2}}(b-z)^{2}} .
\end{gathered}
$$


It is easy to check from Eqs. (4.15)-(4.18) that the reduced action (4.6) is ultravioletfinite close to $z=0, b$, thanks also to azimuthal averaging. This result may seem at first sight surprising, since one expects a quadratic divergence by power counting, due to the appearance of $R^{2}$ in the action (4.6). The cancellation of such terms is due to the choice of solution, leading to the behaviour in Eqs. (4.15), (4.16) such that $a(\bar{a})$ is regular close to $z=b \quad(z=0)$ and $\varphi$ is regular around both. It follows that the scale of $R^{2}$ is set by $b^{2}$, so that the only possible divergence is a logarithmic one, which in turn cancels by azimuthal averaging. Finally, due to the property $\varphi^{(1)}(0)=\varphi^{(1)}(b)=0$, the action is not sensitive to possible $\delta$-function singularities of the fourth derivative (4.18) close to $z=0$ or $z=b$.

On the other hand, for large $|z| \gg b$, we have

$$
\begin{gathered}
a^{(1)} \simeq \bar{a}^{(1)} \simeq \frac{1}{2 \pi b^{2}} \log \frac{|z|}{L}+0\left(\frac{1}{b|z|}\right), \\
\varphi^{(1)} \simeq \frac{2}{\pi^{2}} \frac{\left(z-z^{*}\right)}{b} \log \frac{|z|}{b}+0\left(\left(\log \frac{|z|}{b}\right)^{2}\right) .
\end{gathered}
$$

Thus, the $L$-dependent constant in (4.13) is needed to make $a^{(1)}(L)=0$ (up to terms $\sim b / L$, vanishing in the large- $L$ limit). The rather bad infrared behaviour (4.19) ((4.20)) is forced by the short-distance regularity conditions, but is anyway compensated by the corresponding derivatives (4.17) ((4.18)) in the reduced action. In fact, the integrand of Eq. (4.6) behaves at large distances as $|z|^{-3} \log |z|$ and is thus convergent, as anticipated.

Finally, by introducing $a^{(1)}$ and $\varphi^{(1)}$ in the reduced action, we can calculate the correction of order $R^{2} / b^{2}$ to the leading eikonal. We find, by Eq. (4.6)

$$
\begin{gathered}
A^{(1)}=2 \pi G s\left[\frac{1}{2}\left(\bar{a}^{(1)}(0)+a^{(1)}(b)\right)+\int d^{2} x\left(a^{(0)} \partial^{*} \partial \bar{a}^{(1)}-\bar{a}^{(0)} \partial^{*} \partial a^{(1)}\right)\right. \\
\left.+\left(\frac{\pi R}{2 b}\right)^{2} \frac{1}{2} \int d^{2} x\left(\varphi^{*(1)}\left(\partial^{*} \partial\right)^{2} \varphi^{(1)}+c . c .\right)\right]
\end{gathered}
$$

Noting that the $a^{(0)} a^{(1)}$ interference term is a total derivative we find that the boundary terms cancel altogether, so that the first line of Eq. (4.21) adds up to zero. The second line is explicitly computed by complex integration of the expression resulting from Eqs. (4.14) and (4.18). By using the integrals

$$
\begin{gathered}
\int \frac{d x d y}{2 \pi} \frac{\log z \log \left(1-z^{*}\right)}{z^{*^{2}}(1-z)^{2}}=\frac{\pi^{2}}{6}, \quad \int \frac{d x d y}{2 \pi} \frac{\log z}{z^{*^{2}}(1-z)^{2}}=0, \\
\int \frac{d x d y}{2 \pi} \frac{f(1-z)}{z^{*^{2}}(1-z)^{2}}=-f(1)=\frac{1}{2}\left(1-\frac{\pi^{2}}{6}\right)
\end{gathered}
$$


we obtain

$$
A^{(1)}=G s\left(\frac{R}{b}\right)^{2}, \quad \operatorname{Re} \delta_{2}=\frac{1}{2} \frac{G s}{\hbar}\left(\frac{R}{b}\right)^{2},
$$

in agreement with Eq. (2.10).

The above derivation of $\operatorname{Re} \delta_{2}$ is not trivial, but is anyway easier than a full two-loop calculation and lends itself to a straightforward generalization to higher orders. In fact further iterations of Eq. (4.4) by a power series in $R^{2} / b^{2}$ keep, order by order, the structure of the homogeneous equations (4.11) and thus of the additive arbitrary functions (4.12). The latter can thus be determined at any given order by the single-valuedness and shortdistance conditions $(a)$ and $(b)$ still keeping the infrared behaviour in Eqs. (4.17)-(4.20), apart from an $\frac{R}{b}$-dependent coefficient. It follows that the reduced action in Eq. (4.6) is infrared-safe to all orders.

As a further step, one may look for exact solutions of Eqs. (4.4), subject to the conditions $(a)$ to $(c)$ above, and having an infrared behaviour of the type in Eqs. (4.19) and (4.20), which is perturbatively stable. Although Eqs. (4.4) are not conformal invariant, the solutions are expected to be sums of products of analytic and anti-analytic functions as for $a^{(1)}$ and $\varphi^{(1)}$ in Eqs. (4.13), (4.14). Unfortunately, the $b$-dependence implies a lack of spherical symmetry even in the large- $|z|$ limit, as one can see from Eq. (4.20). Thus finding such exact solutions is expected to be a non-trivial problem, whose detailed analysis is left for future work.

\section{Discussion}

We have proposed a generalized eikonal $S$-matrix for high-energy gravitational scattering in $3+1$ dimensions, based on a regularized effective action that resums all semiclassical terms generated from the superstring approach.

By semi-classical we mean here all terms that are of order $\hbar^{-1}$ in the eikonal phase but contain arbitrary powers of $R^{2} / b^{2}$ at some fixed value of $G s$ (which plays the role of gravitational charge).

The action itself is the one recently proposed by Lipatov ${ }^{(9)}$, apart from surface terms (total derivatives in the effective Lagrangian) that are essential for the (Bloch-Nordsieck) subtraction of infrared divergences.

We have actually checked, by solving perturbatively the classical equations of motion, that the resulting $S$-matrix agrees up to first subleading order (i.e. to order $R^{2} / b^{2}$ ) with the results previously obtained ${ }^{(2)}$ in the diagrammatic approach. 
As compared to the latter, the effective action method allows a much deeper insight into the structure of the (generalized) eikonal phase. For instance, in a simplified treatment in which rescattering of emitted gravitons is neglected, the longitudinal coordinate dependence can be explicitly solved for in terms of a generalized shock-wave. Furthermore, the relevant components of the metric tensor satisfy non-linear equations determined by a reduced action which only involves transverse coordinates.

This new method thus provides directly the dynamically generated metric, and in particular the profile function in the transverse plane. In this sense, it also provides an "off-shell" description of the scattering process that may help interpreting any kind of singularities possibly emerging in the $S$-matrix.

As a matter of fact the classical action defines perturbatively a pure phase, due to the hermiticity properties of the Lagrangian, which can be interpreted as the finite real part of the generalized eikonal, of the form

$$
\operatorname{Re} \delta=\frac{G s}{\hbar} \sigma\left(\frac{R^{2}}{b^{2}}\right)
$$

It is conceivable that when $R / b$ approaches some critical value of order unity, the function $\sigma$ builds up some non-perturbative singularity signalled by infinities and/or unitarity and hermiticity loss.

In order to understand such phenomenon, each one of the abovementioned results will be important. Infrared regularization allows us to disentangle new kinds of inelastic channels from the trivial ones related to bremsstrahlung. Knowledge of the associated metric yields a configuration space picture of the singularity to be compared with the single-parameter behaviour of the $S$-matrix. In this sense we are ready, as claimed, to tackle the description of black-hole formation at Planckian energies.

We are aware, of course that even if such a description were made possible by the present method, it would still be of semiclassical nature. It would suffice, however, to reveal novel effects that extend to distances much larger than the string length where small-distance fluctuations are smeared out. Small-distance details and behaviours will indeed be controlled by quantum effects generated by internal loops for which the string size regularization plays an important role.

Thus the study of non-perturbative solutions of the effective action now appears as an attainable goal to investigate novel "macroscopic" features of a consistent quantum theory of gravity. 


\section{REFERENCES}

(1) D.Amati, M.Ciafaloni and G.Veneziano, Phys. Lett. 197B (1987) 81; Int. J. Mod. Phys. A3 (1988) 1615; Phys. Lett. B 216 (1989) 41.

(2) D.Amati, M.Ciafaloni and G.Veneziano, Nucl. Phys. B347 (1990) 550.

(3) G.'t Hooft, Phys. Lett. 198B (1987) 61.

(4) I.Muzinich and M.Soldate, Phys. Rev. D37 (1988) 353.

(5) See, e.g. R.F.Stark and T.Piran, in Proc. of the 14th Yamada Conference on Gravitational Collapse and Relativity, Kyoto 1986 (H. Sato and T. Nakamura eds., WSPC, Singapore 1986) p.249.

(6) P.D.D'Eath and P.N.Payne, Phys. Rev. D46 (1992)658, 675, 694.

(7) L.N.Lipatov, Sov. Phys. JETP 55 (1982) 582; Nucl. Phys. B307 (1988) 705.

(8) M.Ademollo, A.Bellini and M.Ciafaloni, Nucl. Phys. B338 (1990) 114.

(9) L.N.Lipatov, Nucl. Phys. B365 (1991) 614.

(10) G.Veneziano, Europhys. Lett. 2 (1986) 133; talk presented at the Italian Physical Society Meeting (Naples, 1987);

D.Gross, Proc. Int. Conf. in High Energy Physics, Munich, 1988.

D.Amati, M.Ciafaloni and G.Veneziano, Phys. Lett. B216 (1989) 41;

G.Veneziano, Proc. of Texas Superstring Workshop, 1989;

(11) E.Verlinde and H.Verlinde, Princeton preprint PUPT-1279 (1991).

(12) P.C.Aichelburg and R.U.Sexl, Gen. Rel. Grav. 2 (1971) 303.

(13) Refs. (1), (3), (4) and (9); see also D.Amati, M.Ciafaloni and G.Veneziano, Phys. Lett. B289 (1992) 87. 


\section{FIGURE CAPTIONS}

Fig. 1: $S$-matrix diagram for the multiple scattering series of Eq. (2.6). Crosses denote on-shell lines.

Fig. 2: Regge-Gribov $H$-diagram, embodying the first subleading two-loop corrections; dashed lines denote string gravitons and the vertex is defined in Fig. 3.

Fig. 3: Feynman diagram definition of the Regge-Gribov vertex of Fig. 2, including all external line insertions.

Fig. 4: Structure of $2 n$-loop diagrams contributing irreducible semiclassical terms to the effective eikonal.

Fig. 5: (a) Emission and (b,c) rescattering vertices of intermediate gravitons (wavy lines) by Regge-Gribov gravitons (dashed lines) 


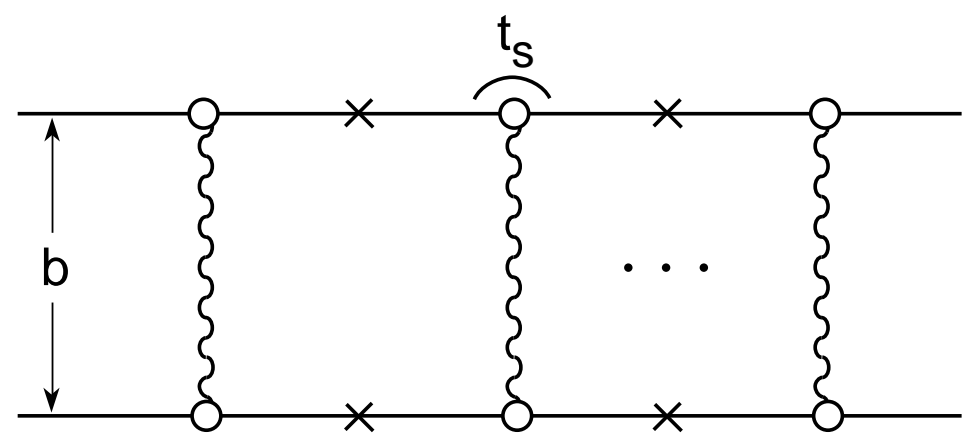

Fig. 1

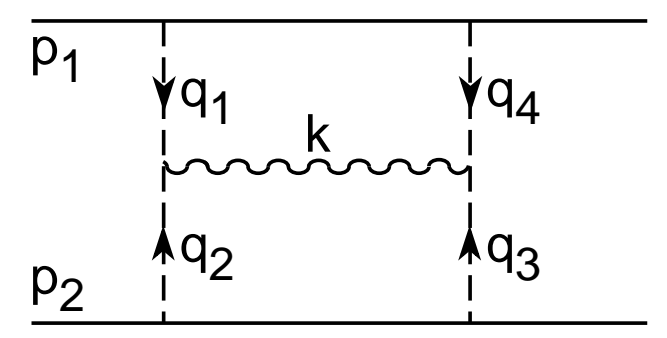

Fig. 2 


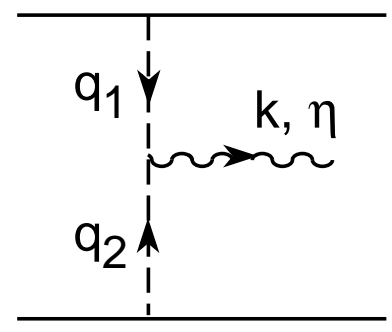

a)

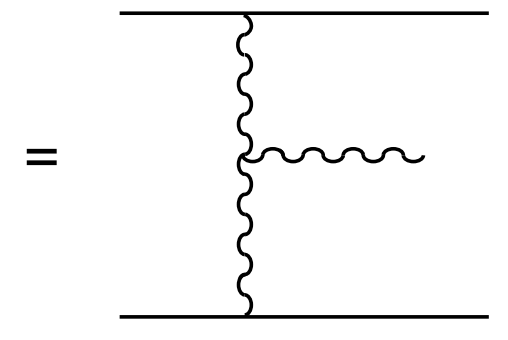

b)

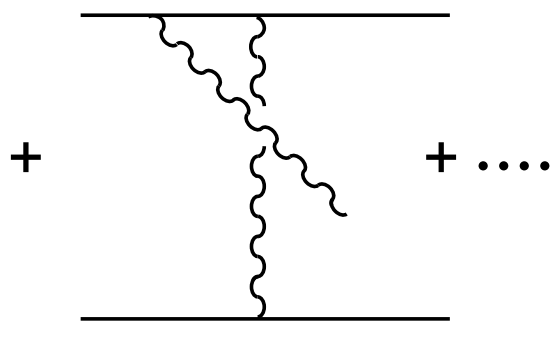

c)

Fig. 3

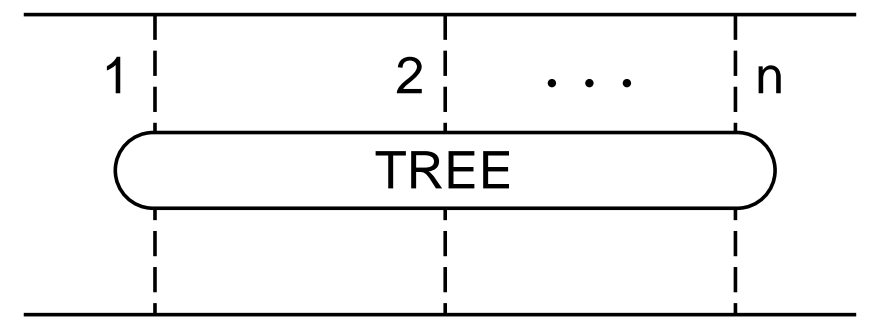

Fig. 4
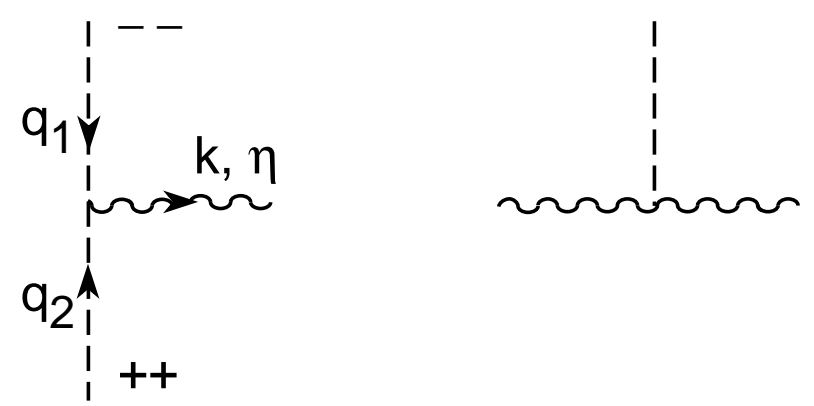

a)

b)

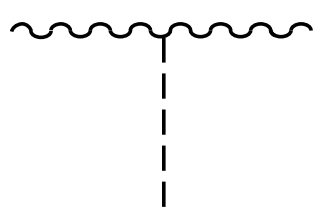

c)

Fig. 5 